\title{
OBSERVATIONS DES POPULATIONS D'ABEILLES ET DE VARROA DANS LES COLONIES À DIFFÉRENTS NIVEAUX D'INFESTATION
}

\author{
W. RITTER *, E. LECLERCQ ** et W. KOCH* \\ * Tierhygienisches Institut, Am Moosweiher 2, D 7800 Freiburg i. Br. \\ *:Ecole supérieure d'agriculture de Purpan, 271, av. de Grande-Bretagne \\ F 31016 Toulouse Cedex
}

\begin{abstract}
RÉSUME
De nombreuses colonies ont été décimées par la Varroatose. Le processus qui aboutit à l'effondrement des colonies sera présenté à l'aide des facteurs suivants : population d'abeilles et infestation de Varroa. L'évolution de ces facteurs a été analysée toutes les 3 semaines de mai à septembre. La surface du couvain et le nombre d'abeilles des colonies fortement infestées a chuté de façon dramatique à partir de juillet. Ces événements conduisirent à la disparition de la colonie. Dans les colonies effondrées on a pu remarquer la présence du APVirus dans les abeilles adultes et une décomposition du couvain provoquée par des bactéries.
\end{abstract}

\section{INTRODUCTION}

En République fédérale Allemande, comme dans d'autres pays, le nombre de colonies infestées par Varroa est en constante augmentation. En Allemagne du Sud, l'année 1982 fut marquée par l'apparition des premiers cas de collapsus dépeuplant, dans la plupart des ruches non traitées. Les apiculteurs ne constatèrent souvent les dégâts, qu'après que les colonies aient été déjà très affaiblies. Avant ce collapsus, aucun changement de l'état des colonies - tel que diminution de récolte, altération du comportement, symptômes cliniques variés, etc. n'avait été constaté.

Population d'abeilles et degré d'infestation devaient être mesurés afin de pouvoir analyser quantitativement le processus qui aboutit au collapsus dépeuplant. Cependant, la détermination de la population de Varroa à l'aide d'une appréciation par calcul ne fut pas utilisée. Les modèles existants nous auraient conduits à de trop grandes erreurs d'interprétation (FuCHS et KoENIGER, 1984). 


\section{MATERIEL ET METHODE}

De la mi-mai à début septembre, nous avons déterminé la population d'abeilles ainsi que celles de Varroa dans trois groupes comprenant chacun deux colonies. Ces trois groupes étaient situés dans la région de Fribourg-en-Brisgau (RFA). Un groupe était constitué de colonies qui, au début, n'étaient pas infestées ou très faiblement infestées. Les deux autres groupes étaient formés de colonies qui étaient moyennement à fortement infestées.

Toutes les trois semaines, les surfaces de couvain operculé et ouvert, ainsi que le nombre d'abeilles, furent estimés selon une méthode mise au point par Gerig (1983). Simultanément à ces mesures, furent prélevés des échantillons d'abeilles adultes et de couvain operculé, ceci pour déterminer la population de Varroa. Les échantillons de couvain furent examinés en laboratoire (Ritter, 1980 ; Perschil : vom Hoevel, 1983). Chaque semaine des feuilles de papier installées sur le plateau permirent de recueillir et de compter les Varroa tombés naturellement.

\section{RESULTATS}

Au début de l'essai (fin mai), la population des colonies du groupe faiblement infesté était en moyenne de 18000 abeilles, celle du groupe moyennement infesté de 11000 et celle du groupe fortement infesté de 15000 abeilles (Fig. 1). Dans le groupe faiblement infesté, la population dépasse 30000 abeilles à la fin juillet. Ensuite, on constate une diminution insignifiante mais constante, jusqu'à 29000 abeilles au début septembre. Au contraire, la force des colonies du groupe moyennement infesté n'augmente seulement que jusqu'au début juillet. Dans le groupe fortement infesté, elle n'augmente que jusqu'à mi-juin. Ensuite la force des colonies dans ces deux groupes diminue si rapidement que les colonies du groupe moyennement infesté n'avaient plus que 2500 abeilles début septembre et que celles du groupe fortement infesté étaient déjà mortes.

Si on compare le développement de la population d'abeilles au degré d'infestation des abeilles adultes, en prenant l'exemple du groupe fortement infesté, on constate une corrélation évidente entre ces deux facteurs.

Plus la population chute, plus - comme on pouvait le prévoir - l'infestation relative des abeilles adultes augmente (Fig. 2). Cette augmentation de l'infestation des abeilles adultes est particulièrement remarquable, puisqu'elle est passée de $18 \%$ fin juillet à $78 \%$ mi-août. Parallèlement, la force de la colonie avait chuté de 6300 à 1200 abeilles. Le nombre total de Varroa parasitant les abeilles adultes était resté constant ou n'avait que légèrement diminué. Des faits semblables furent également observés dans le groupe moyennement infesté. Au contraire, le niveau d'infestation est resté faible dans les colonies faiblement infestées. 


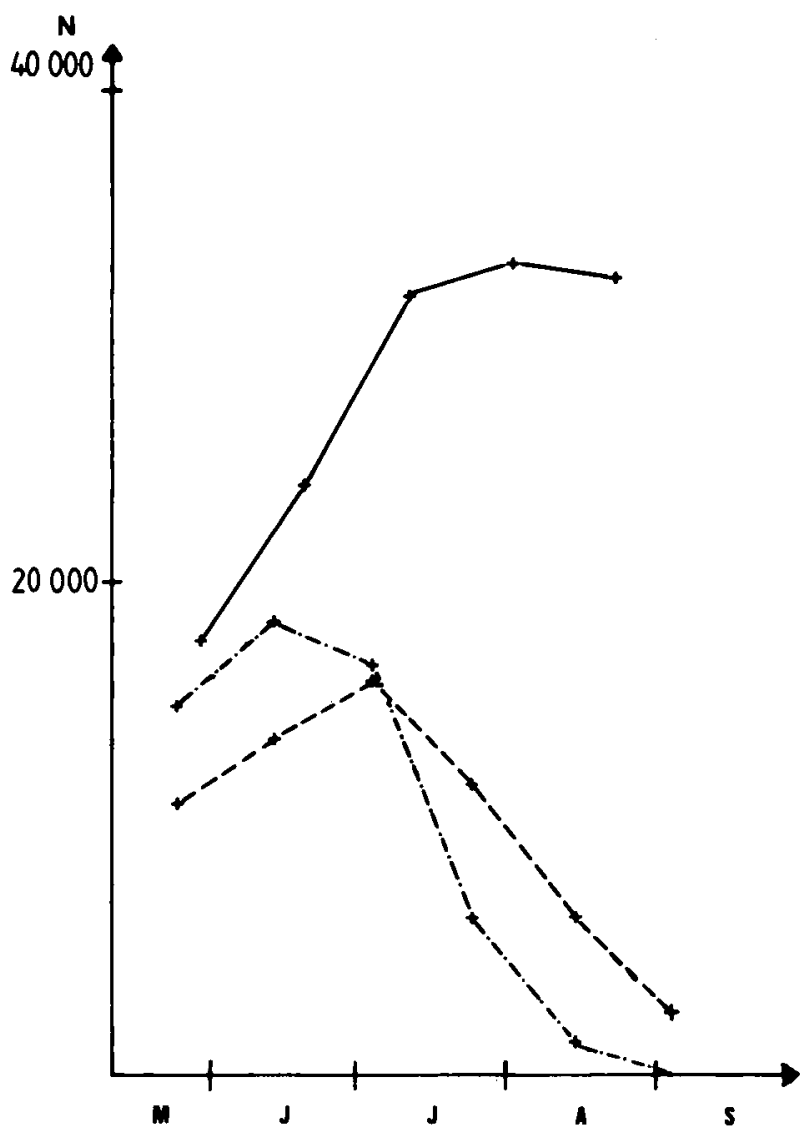

FIG. 1. - Population d'abeilles de ruches à différents niveaux d'infestation En ordonnées : $\mathbf{N}=$ nombre d'abeilles

En abscisses : mois de l'année

\section{colonies faiblement infestées}

- - - colonies moyennement infestées

$-\ldots \cdot-.-$ colonies fortement infestées

FIG. 1. - Bee population of colonies at different levels of infestation

$\mathrm{Y}$-axis : $\mathrm{N}=$ hee population

$\mathrm{X}$-axis : months

slightly infested colonies

moderately infested colonies

$-\cdot-\ldots$ highly infested colonies 


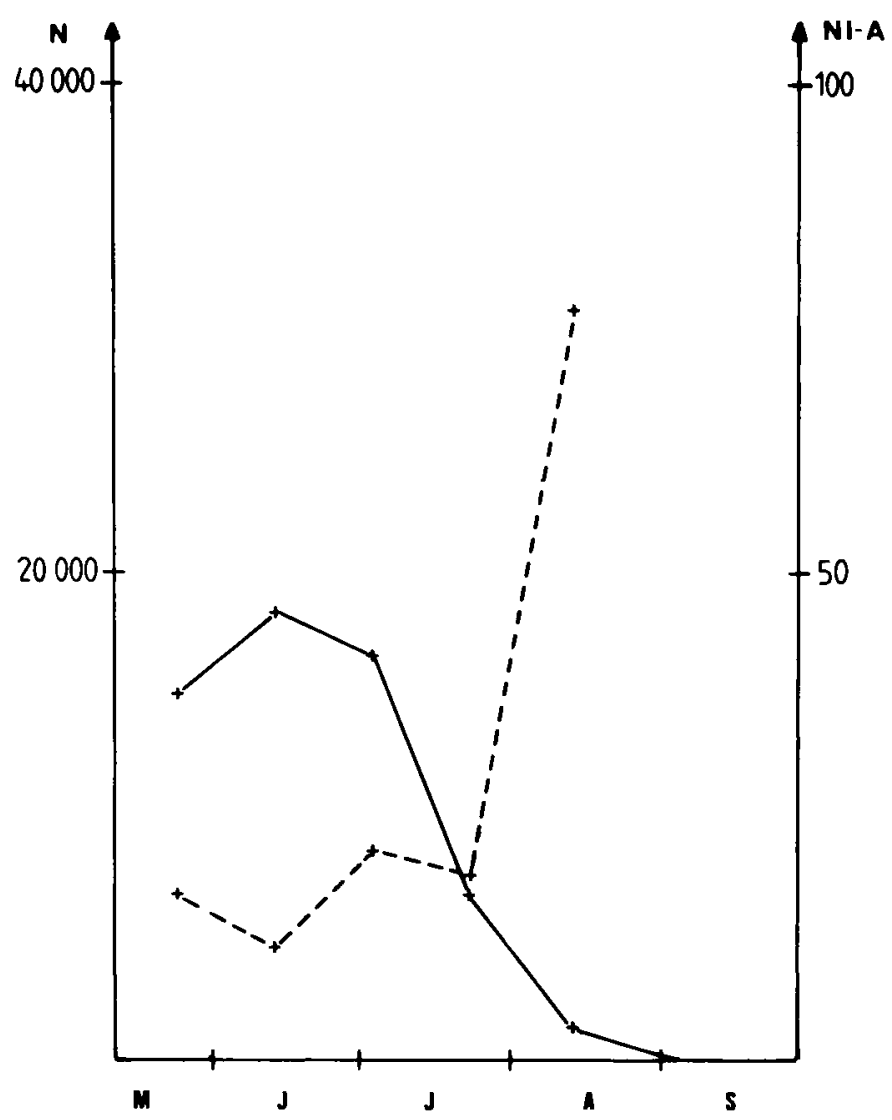

FIG. 2. - Population d'abeilles et leur niveau d'infestation dans des ruches fortement infestées En ordonnées :

$\mathbf{N}=$ nombre d'abeilles

- - - NI-A = niveau d'infestation des abeilles (\%)

Fig. 2. - Bee population and its degree of infestation in highly infested colonies Y-axis :

$\mathbf{N}=$ bee population

NI-A $=$ degree of bee infestation $(\%)$

A l'aide du rapport estimé :

nb de jour de vie (j) $\times$ nb d'abeilles (n)

nb de cellules de couvain ( $n$ )

on obtient la durée de vie moyenne des abeilles (j) (BüHLmann, 1984). Pendant la période d'essai, de mai à septembre, la durée moyenne de vie des abeilles était dans le groupe faiblement infesté de 15,6 jours, dans le groupe moyennement infesté de 9,1 jours et dans le groupe fortement infesté de 8,3 jours. Chute de la 
durée de vie des abeilles et degré d'infestation, sont deux phénomènes simultanés. Les données obtenues en laboratoire par DE JoNG (1984) ont ainsi pu être vérifiées en conditions naturelles. En outre on a pu identifier l'A.P.V. (Acute Paralysis Virus) aussi bien dans les ruches moyennement infestées que fortement infestées. Il reste encore à savoir dans quelle mesure ce virus agit de façon pathogène, sur les abeilles adultes.

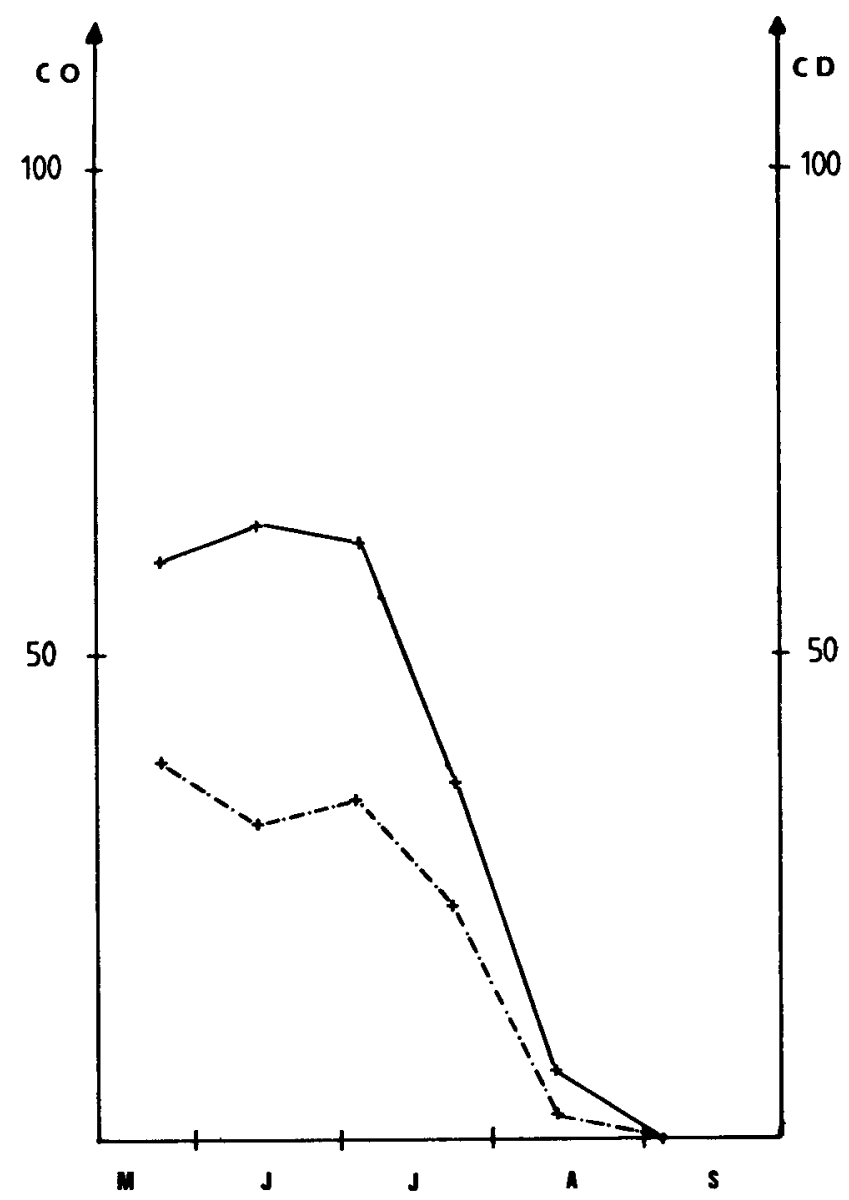

FIG. 3. - Surface de couvain operculé/surface de couvain non operculé dars des ruches fortement infestées

En ordonnécs :

CO = surface de couvain operculé $\left(\mathrm{dm}^{2}\right)$

$\mathrm{CD}=$ surface de couvain non operculé $\left(\mathrm{dm}^{2}\right)$

Fig. 3. - Sealed brood area/unsealed brood area in highly infested colonies Y-axis : 
Les phénomènes observés sur le couvain operculé et ouvert sont semblables à ceux décrits précédemment en ce qui concernait les abeilles. La figure 3 , relative au groupe fortement infesté, met bien en évidence cette évolution parallèle. Pendant la période de collapsus dépeuplant, la surface de couvain operculé et ouvert chuta de manière brutale. Le couvain operculé diminua relativement plus vite que le couvain ouvert. Jusqu'à juillet, la part du couvain operculé dans la surface totale de couvain (couvain operculé et ouvert) était beaucoup plus grande que lors de l'effondrement.

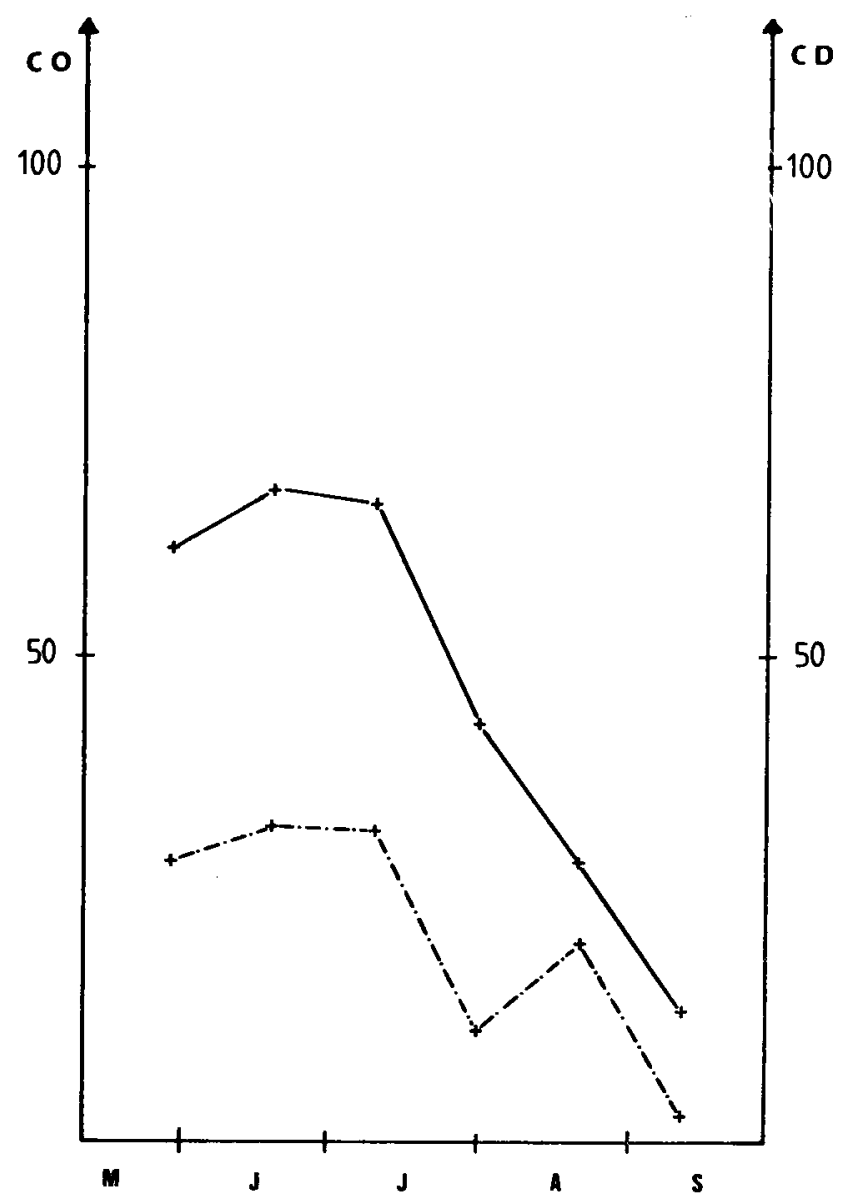

Fig. 4. - Surface de couvain operculé/surface de couvain non operculé dans des ruches faiblement infestées

En ordonnées :

$$
\mathrm{CO}=\text { surface de couvain operculé }\left(\mathrm{dm}^{2}\right)
$$

$-.-.-\mathrm{CD}=$ surface de couvain non operculé $\left(\mathrm{dm}^{\prime}\right)$

FIG. 4. - Sealed brood area/unsealed brood area in slightly infested colonies Y-axis :

$$
\text { - } \mathrm{CO}=\text { sealed brood area }\left(\mathrm{dm}^{2}\right)
$$

$-\cdot \ldots+-\mathrm{CD}=$ unsealed brood area $\left(\mathrm{dm}^{2}\right)$ 
La chute plus rapide du couvain operculé peut s'expliquer :

- soit par le fait que les abeilles abandonnent le couvain plus âgé et fortement infesté ;

- soit par le fait que le couvain périt en raison d'une refroidissement provoqué par une réduction de la force de la colonie.

Les surfaces de couvain operculé et ouvert diminuent davantage en raison d'une attaque microbienne. Cette attaque présente des symptômes cliniques très proches de ceux de la loque européenne. Les mêmes observations ont été réalisées sur le groupe des colonies moyennement infestées.

Dans les colonies faiblement infestées, l'état du couvain ainsi que le rapport des surfaces de couvain operculé et ouvert était «normal» (Fig. 4). Alors que dans les colonies faiblement infestées, l'infestation du couvain était minime, elle augmenta dans les colonies moyennement et fortement infestées pendant la période d'observation.

Grâce à l'exemple du groupe fortement infesté (Fig. 5), on note que pendant la période du collapsus dépeuplant, de fin juillet à mi-août, l'infestation relative du couvain a plus que doublé. La plupart des cellules sont alors occupées par 2 et le plus souvent même par 3 Varroa. Ceci ne doit pas nous conduire à la conclusion que le nombre total de Varroa a augmenté : en effet, le nombre de $V$ arroa restant constant, la surface de couvain diminuant, le niveau relatif d'infestation doit augmenter dans la même proportion, ainsi qu'on l'a montré.

La mortalité naturelle de Varroa est une façon de suivre l'évolution de la population du parasite. En juillet, dans le groupe fortement infesté sont morts dans chaque ruche presque 1600 Varroa par semaine (Fig. 6), tandis que dans le groupe moyennement infesté, cette valeur n'a été atteinte que fin août. Le développement de la parasitose est donc identique dans les deux groupes, il ne se déroule qu'avec un mois de retard dans le groupe moyennement infesté.

La rédaction de cet article est basée sur l'analyse des données et d'observations effectuées lors d'un programme d'expérimentation beaucoup plus important. II s'est déroulé de mi-mai à fin octobre 1984 à l'Institut Vétérinaire de Fribourgen-Brisgau (RFA). Il fera l'objet de la rédaction d'un mémoire de fin d'études à l'Ecole Supérieure d'Agriculture de Purpan à Toulouse (France).

Reçu pour publication en septembre 1984. 


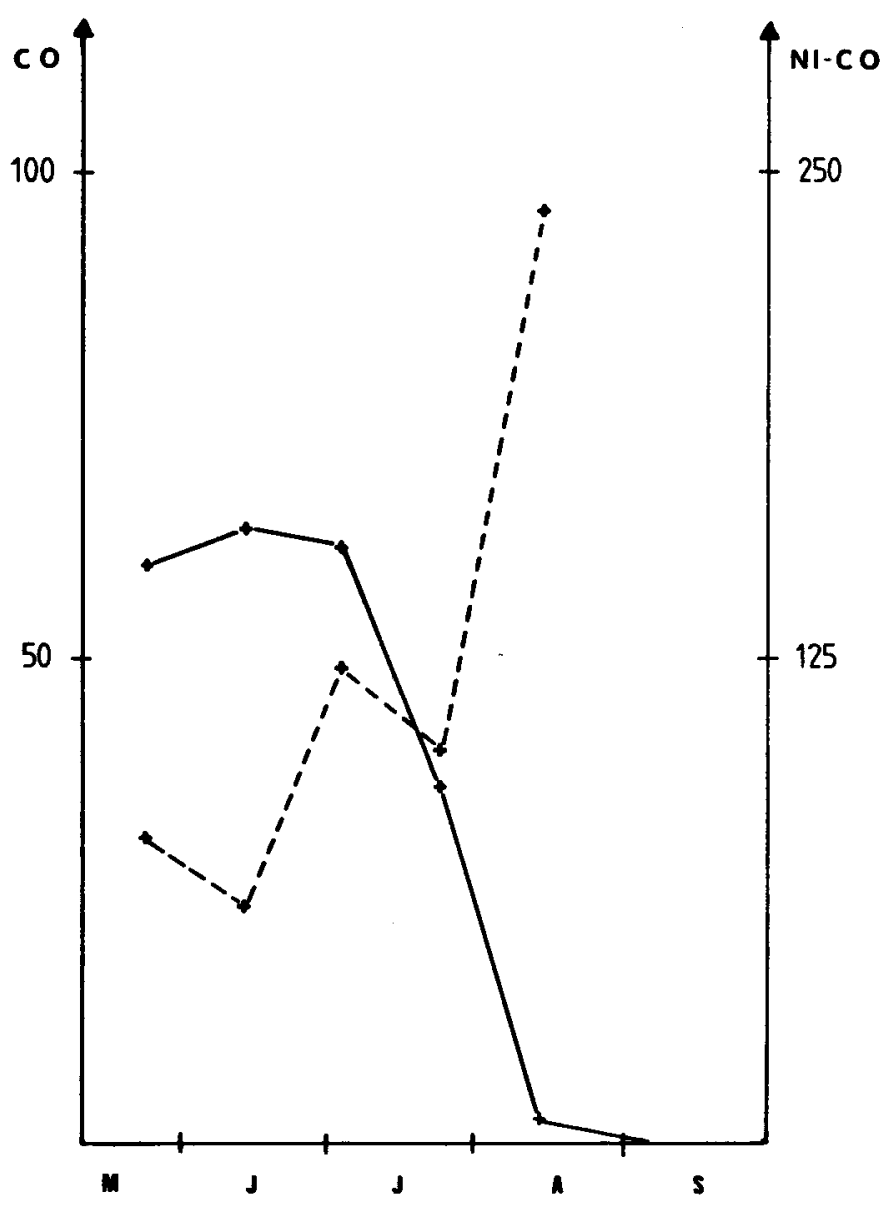

FIG. 5. - Surface de couvain operculé et son niveau d'infestation dans des ruches forfement infestées En ordonnées :

$\mathrm{CO}=$ surface de couvain operculé $\left(\mathrm{dm}^{2}\right)$

- - NI-CO = niveau d'infestation du couvain operculé $(\%)$

FIG. 5. - Sealed brood area and its degree of infestation in highly infested colonies Y-axis : $\mathrm{CO}=$ sealed brood area $\left(\mathrm{dm}^{2}\right)$

$--1 \%$ NI-CO $=$ degree of sealed brood infestation $(\%)$ 


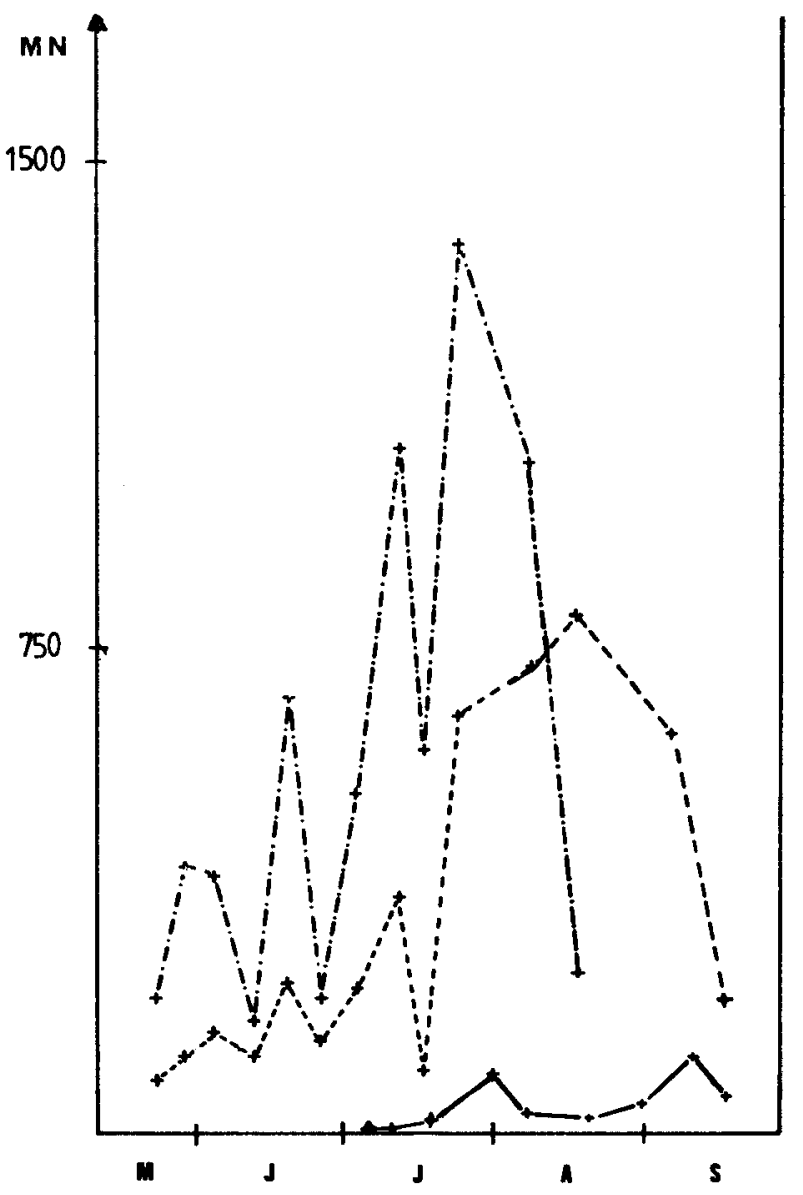

Fig. 6. - Mortalité naturelle de Varroa dans des colonies moyennement et fortement infestées En ordonnées : MN = mortalité naturelle (en nombre d'abeilles) Mêmes légendes que Fig. 1

FIG. 6. - Natural mite mortality in moderately and highly infested colonies $\mathrm{Y}$-axis : $\mathrm{MN}=$ natural mortality (in number of bees) Legends as in Fig. 1 


\section{SUMMARY \\ OBSERVATIONS ON BEE AND VARROA MITE POPULATIONS IN INFESTED HONEY BEE COLONIES}

\section{Introduction}

Numerous bee colonies in Germany, as well as, in other countries have died of varroatosis. The events up to the point of collapse of the colonies can be demonstrated with the bee population and the degree of mite infestation.

\section{Methods}

The bee and mite populations in 6 colonies were calculated from mid May until the beginning of September. On this basis the change in populations and parasitic development was to be observed up to the collapse of the bee colonies. The colonies studied were divided into three groups : The colonies of the first group had relatively few mites at the start of the experiment. The 2 nd group was well infested and the 3rd strongly. Nevertheless all colonies were in good shape at the start of the experiment in May. The bee population was calculated in three week intervals by counting the number of natural mite mortalities as well as, extrapolating from infestation in adult bees and sealed brood.

\section{Results and discussion}

The mildly-infested colonies had 18000 bees on the average, the middle-infested colonies 11000 bees and the strongly-infested 15000 bees at the start of the experiment at the end of May. Whereas the population increased in the mildly-infested colonies, the middle- and strongly-infested decreased to a number of 2500 bees by the beginning of September. The brood decreased in the same manner as the bee population by the end of July. At the same time the degree of infestation of the brood, as well as the bees, increased drastically. The natural death of the mites increased, per colony, on the average of 1500 mites a week. The acute paralysis virus (APV) could be identified in adult bees of the collapsed colonies. The brood of these colonies exhibited clinical symptoms of European Foulbrood.

\section{ZUSAMMENFASSUNG}

\section{UNTERSUCHUNGEN DER BIENEN- UND VARROAMILBEN-POPULATIONEN BEFALLENER VÖLKER DER HONIGBIENE}

\section{Einleitung}

In der Bundesrepublik Deutschland wie in anderen Ländern sind zahlreiche Völker an der Varroatose eingegangen. Die Vorgänge bis zum Zusammenbruch der Völker sollten anhand der Bienenpopulation und des Milbenbefalls dargestellt werden.

\section{Methoden}

In 6 Bienenvölkern wurde die Bienen- und Milbenpopulation von Mitte Mai bis Anfang September ermittelt. Auf dieser Basis wurden Populationsveränderungen und die Parasitenentwicklung bis zum Völkerzusammenbruch untersucht. Die beobachteten Völker wurden in drei Gruppen eingeteilt. Die Völker der ersten Gruppe waren mit relativ wenigen Milben zu Versuchsbeginn befallen. Die zweite Gruppe war mittelstark und die dritte Gruppe stark infiziert. Alle Völker waren jedoch zu Beginn der Untersuchungen im Mai in einem guten Zustand. Die Bienenpopulation wurde in einem dreiwöchigen Abstand durch Messen der Brutfläche und Zählen der Bienen ermittelt. Die Milbenpopulation wurde aus dem natürlichen Milbenabfall sowie durch Extropolieren des Befalls adulter Bienen und gedeckelter Brut errechnet. 


\section{Ergebnis und Diskussion}

Zum Versuchsbeginn Ende Mai enthielten die schwach befallenen Völker im Durchschnitt 18000 , die mittelstark befallenen 11000 und die stark befallenen 15000 Bienen. Während in den schwach befallenen Völkern die Volkstärke bis Anfang September zunahm, waren die mittelstark befallenen Völker auf 2500 Bienen zurückgegangen und die stark befallenen eingegangen. In gleicher Weise wie die Bienenpopulation ab Ende Juli abnahm, ging auch die Brut zurück. Gleichzeitig nahm der Befallsgrad sowohl der Brut als auch der Bienen drastisch zu. Der natürliche Milbenabfall war im Juli im Durchschnitt pro Volk auf über 1500 Milben in der Woche angestiegen. In adulten Bienen zusammenbrechender Völker konnte das Acute Paralysis Virus (APV) nachgewiesen werden. Die Brut dieser Völker zeigte klinische Symptome der Europäischen Faulbrut.

\section{REFERENCES}

De Jong D., De Jong P.H. and Goncalves L.S., 1982. - Weight loss and other damage to developing worker honey bees due to infestation with Varroa jacobsoni. J. apic. Res., 21 (3), 165-167.

Fuchs S. und Koeniger N., 1984. - Rechnen oder Raten : das Dilemna bei der Abschätzung des Varroabefalles. Allg. dtsch. Imkerztg., 18 (9), 294-296.

Bühlmann G., Gerig L., vom Hövel R., Müller B., Perschil F., Ritter W. und Wille H., 1984. Populationsdynamische Untersunchungen an Varroa-befallenen freifliegenden Bienenvölkern in Baden-Württemberg. Apidologie, 15 (3), 252-254.

Gerig L., 1983. - Lehrgang zur Erfassung der Volksstärke. Schweiz. Bienenztg., 106 (4)، 199-204.

RitTer W., 1980. - Zur Methodik der Prüfung von Chemotherapeutika zur Bekämpfung der Varroatose der Honigbiene. Apidologie, 11 (2), 131-141.

Ritter W., Perschil F. und vom Hövel R., 1983. - Erkennen der Varroatose mit einfachen Untersuchungsmethoden. Allg. dtsch. Imkerztg., 17 (7), 221-222. 